\title{
Leitthema
}

Bundesgesundheitsbl $2017 \cdot 60: 728-736$

DOI 10.1007/s00103-017-2561-6

Online publiziert: 17. Mai 2017

(c) Der/die Autor(en) 2017. Dieser Artikel ist eine Open-Access-Publikation.

CrossMark

Birgit Dusemund' · Ivonne M. C. M. Rietjens ${ }^{2} \cdot$ Alexander Cartus $^{3}$. Bernd Schaefer ${ }^{1} \cdot$ Alfonso Lampen ${ }^{1}$

'Bundesinstitut für Risikobewertung, Berlin, Deutschland

${ }^{2}$ Division of Toxicology, Wageningen University, Wageningen, Niederlande

${ }^{3}$ Lebensmittelchemie und Toxikologie, Technische Universität Kaiserslautern, Kaiserslautern, Deutschland

\section{Pflanzliche Kontaminanten in Lebensmitteln}

\section{Vorkommen, Wirkung und Risikobewertung}

\section{Einleitung}

Seit der Antike sind Intoxikationen bekannt, die durch Kontamination von Lebensmitteln mit pflanzlichen Giften bedingt sind. Beispielsweise beschrieb schon Xenophon in der „Anabasis“ [1], dass sich Soldaten durch den Verzehr eines von Rhododendron ponticum stammenden Honigs vergifteten. Auch heute noch wird in der Fachliteratur über Vergiftungsfälle berichtet, die durch die Verunreinigung von Lebensmitteln mit bestimmten Phytotoxinen hervorgerufen werden, wie z.B. durch Verzehr von mit Grayanotoxinen verunreinigtem Rhododendronhonig [2], eines mit Delta-9-Tetrahydrocannabinol (THC) kontaminierten Hanfsamenöls [3] oder von Mohnsamen, die mit Morphin und Codein verunreinigt waren [4].

In Übereinstimmung mit der Definition von Schrenk [5] werden unter pflanzlichen Kontaminanten hier Substanzen verstanden, die weder natürlich in den Rohstoffen der Lebensmittelherstellung vorkommen noch während des regulären Produktionsprozesses hinzugefügt werden. Als Beispiel für pflanzliche Kontaminanten, gegen deren Aufnahme aufgrund ihrer genotoxischen Kanzerogenität Bedenken bestehen, werden im Folgenden 1,2-ungesättigte Pyrrolizidinalkaloide (PA) beschrieben. Tropanalkaloide (TA), Opiumalkaloide und Delta-9-Tetrahydrocannabinol (THC) stellen hingegen Kontaminanten dar, bei denen unerwünschte akut toxische Effekte im Vordergrund der Risikobewertung stehen, für die Schwellenwerte angenommen werden können. Weitere Beispiele und Einzelheiten zur Risikobewertung pflanzlicher Lebensmittelkontaminanten können anderen Übersichtsartikeln entnommen werden [6-10].

\section{1,2-ungesättigte Pyrrolizidin- alkaloide (PA) \\ Chemische und botanische Charakterisierung, Vorkommen}

1,2-ungesättigte Pyrrolizidinalkaloide (PA) sind sekundäre Pflanzenstoffe mit mehr als 660 einzelnen Vertretern sowie den jeweiligen $\mathrm{N}$-Oxiden, wobei aufgrund chemotaxonomischer Überlegungen in über 6000 Pflanzenspezies mit deren Vorkommen gerechnet wird. Vornehmlich gehören PA-haltige Pflanzen den Familien der Asteraceae (z. B. die Gattungen Senecio, Eupatoria, Tussilago), der Boraginaceae (z. B. die Gattungen Heliotropium, Symphytum, Trichodesma) und der Fabaceae oder Leguminosae (z. B. die Gattung Crotalaria) an [11, 12].

Pyrrolizidinalkaloiden mit 1,2-ungesättigter Necinstruktur(PA), die mit mindestens einer verzweigten C5-Carbonsäure (Position 9) verestert sind, werden hepatotoxische, karzinogene und mutagene Wirkungen zugeschrieben, wobei das Vorhandensein einer weiteren Hydroxygruppe in Position 7 und deren einfache oder zyklische Veresterung jeweils mit einer Wirkungsverstärkung assoziiert wird. Die beispielhaft in 0 Abb. 1 dargestellten PA kommenu. a. in Symphytum

\begin{tabular}{|c|c|}
\hline \multicolumn{2}{|c|}{ Abkürzungen } \\
\hline$A R f D$ & Akute Referenzdosis \\
\hline$B f R$ & Bundesinstitut für Risikobewertung \\
\hline$B g V V$ & $\begin{array}{l}\text { Bundesinstitut für gesundheit- } \\
\text { lichen Verbraucherschutz und } \\
\text { Veterinärmedizin }\end{array}$ \\
\hline$B M D L_{10}$ & $\begin{array}{l}\text { Benchmark Dose Level, unteres Kon- } \\
\text { fidenzlimit mit einer Responserate } \\
\text { von } 10 \%\end{array}$ \\
\hline COT & $\begin{array}{l}\text { Committee on Toxicity of Chemicals } \\
\text { in Food, Consumer Products and the } \\
\text { Environment (UK) }\end{array}$ \\
\hline EFSA & European Food Safety Authority \\
\hline$F A O$ & $\begin{array}{l}\text { Food and Agriculture Organization of } \\
\text { the United Nations }\end{array}$ \\
\hline IARC & $\begin{array}{l}\text { International Agency for Research on } \\
\text { Cancer }\end{array}$ \\
\hline JECFA & $\begin{array}{l}\text { Joint FAO/WHO Expert Committee } \\
\text { on Food Additives }\end{array}$ \\
\hline KG & Körpergewicht \\
\hline LOAEL & $\begin{array}{l}\text { Lowest Observed Adverse Effect } \\
\text { Level }\end{array}$ \\
\hline LOEL & Low effect Level \\
\hline$M O E$ & Margin of Exposure \\
\hline NOAEL & No Observed Adverse Effect Level \\
\hline$P A$ & $\begin{array}{l}\text { 1,2-ungesättigte Pyrrolizidinalkaloi- } \\
\text { de }\end{array}$ \\
\hline$T A$ & Tropanalkaloide \\
\hline THC & Delta-9-Tetrahydrocannabinol \\
\hline$H V O D$ & Hepatic veno-occlusive disease \\
\hline WHO & World Health Organization. \\
\hline
\end{tabular}




\section{Zyklische Diester}

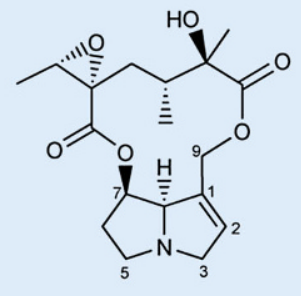

Jacobin

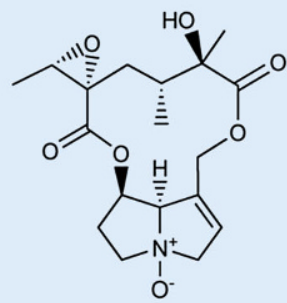

Jacobin-N-Oxid

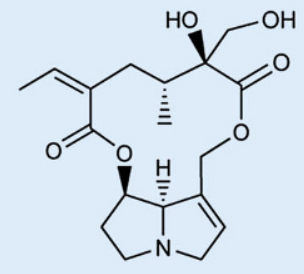

Retrorsin

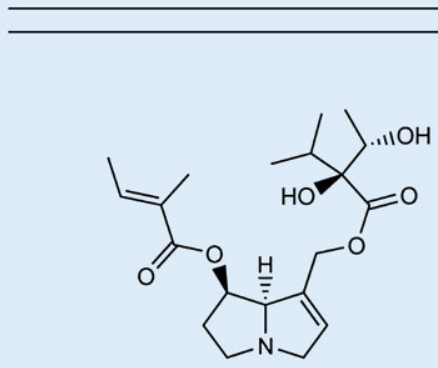

Symphytin

Diester

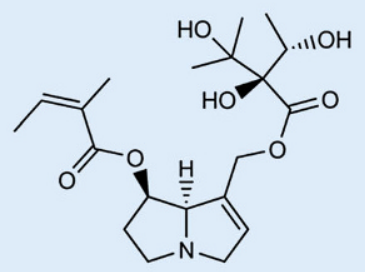

Echimidin

Monoester

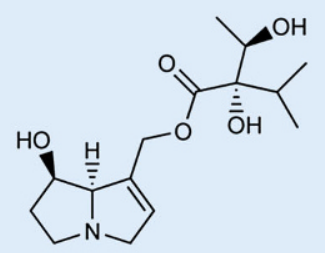

Intermedin

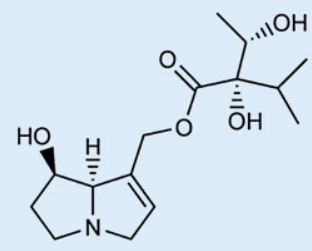

Lycopsamin

Abb. 1 ॥ Strukturformeln einiger Pyrrolizidinalkaloide

officinale (Symphytin, Echimidin, Intermedin, Lycopsamin) oder in Senecio-Arten (Retrorsin, Jacobin) vor.

PA können durch Verunreinigungen mit PA-bildenden Wildkräutern in den Anbauflächen von Nutzpflanzen in Lebensmittel gelangen [13]. In Deutschland sind z. B. Verunreinigungen bei Salaten mit PA-haltigem Kreuzkraut/Greiskraut aufgetreten [14]. Auch die PA-Belastung von Tee und Kräutertees wird auf eine Kontamination bei der Ernte mit PAbildenden Wildkräutern in den Anbauflächen zurückgeführt $[15,16]$. Bienenprodukte wie Honig und Pollen können ebenfalls durch PA belastet sein, wobei PA-bildende Wildpflanzen wie Echium-, Senecio- und Borago-Arten die Kontaminationsquelle darstellen [12, 17].

\section{Gefährdungspotenzial/ toxikologische und pharma- kologische Daten}

Die akute und chronische Lebertoxizität von PA beim Menschen ist durch Vergiftungsfälle nach arzneilicher Einnahme von PA-haltigen Kräutertees und -erzeugnissen sowie Berichten über epidemische Lebererkrankungen mit Todesfällen in Pakistan, Indien, Afghanistan, Tadschikistan und der UdSSR nach Verzehr von Getreide, das mit Samen von Heliotropium- oder CrotalariaArten kontaminiert war, belegt (z. B. $[11,18])$. Das gehäufte Auftreten von Leberzirrhosen bei Schlachtrindern, die Alpenkreuzkraut (Senecio alpinus) mit Heu und Silage gefressen hatten, sowie die mit Leberdegeneration einhergehen- den Seneciosen, die bei Pferden nach Aufnahme von Senecio-Arten beim Weiden beschrieben wurden, sind Beispiele aus der Tierhaltung (z. B. [11]).

Die in der Pflanze neben freien PA vorliegenden PA- $\mathrm{N}$-Oxide haben bei oraler Applikation die gleiche Toxizität wie die reduzierte Form der Alkaloide, zu der sie durch die Reduktasen des Darmes metabolisiert werden. Die Verstoffwechselung der PA erfolgt durch Hydrolyse, $\mathrm{N}$-Oxidation und Dehydrogenierung des Pyrrolizidinrings. Die bei der Hydrolyse frei werdenden Necinsäuren scheinen toxikologisch keine Relevanz zu besitzen. Gebildete N-Oxid-Metaboliten sind sehrgut wasserlöslich und werden schnell mit dem Urin ausgeschieden, sodass dieser Stoffwechselweg als Entgiftung betrachtet wird. Die in der Leber durch Cytochrom-P450-Monooxygenasen katalysierte Dehydrogenierung des Pyrrolrings der ungespaltenen Ester zu toxischen Pyrrolestern stellt jedoch eine Giftungsreaktion dar. Pyrrole dieses Typs sind als hochreaktive alkylierende Agenzien in der Lage, mit nukleophilen Gruppen von Nucleinsäuren und Proteinen zu Addukten zu reagieren, und werden als aktive Metaboliten angesehen, die die hepatotoxischen, hepatokarzinogenen und genotoxischen Wirkungen der PA bedingen (z. B. $[6,11,19])$.

Die Leber ist das primäre Zielorgan PA-bedingter Schädigungen, jedoch können auch andere Organe wie die Lunge betroffen sein. Insgesamt sind hinsichtlich der toxischen Effekte nach Aufnahme ungesättigter PA nichtneoplastische Schäden und genotoxischkanzerogene Wirkungen unterscheidbar.

Als nichtneoplastische Schäden treten bei Mensch und Tier Leberschädigungen auf, die zu Lebernekrosen führen können. Typisch sind venookklusive Veränderungen in der Leber („hepatic veno-occlusive disease“, HVOD), aber auch in der Lunge, wobei in der Leber eine Manifestation als Verschluss der zentralen sublobulären Lebervenen erfolgt [20]. Diese toxischen Effekte treten bei der Aufnahme von PA in größeren Dosen innerhalb kurzer Zeit auf, in niedrigeren Dosen nach längerer Zeit. Die für die chronische Lebertoxizität ungesättigter PA beim Tier charakte- 
ristischen vergrößerten Hepatozyten mit großen, hyperchromatischen Zellkernen werden als morphologische Manifestation des antimitotischen Effektes ungesättigter PA angesehen. In höheren Dosen können PA entwicklungstoxische Effekte zeigen [11].

Im Tierversuch wird die kanzerogene Wirkung bestimmter PA als gesichert angesehen und ein entsprechendes Risiko für den Menschen in Betracht gezogen. So wurden Lasiocarpin, Riddelliin und Monocrotalin von der IARC (International Agency for Research on Cancer) in die Gruppe 2B („possibly carcinogenic to humans") eingestuft [21-23]. Bei anderen ungesättigten PA deuten die Tierstudien mit der Verbindung selbst oder ihren aktiven Metaboliten ebenfalls auf eine kanzerogene Wirkung hin, jedoch ist hier die Datenlage unvollständig. Isatidin, Retrorsin und Senkirkin ordnete die IARC der Gruppe 3 („not classifiable“) $\mathrm{zu}$ [21-23].

Vielfach zeigten Verbindungen, die sich im Tierversuch als kanzerogen erwiesen, auch positive Resultate bei der Mutagenitätstestung (z. B. [11, 24]).

\section{Risikocharakterisierung und -bewertung}

Für die Risikobewertung sind insbesondere Studien zur chronischen Toxizität an Ratten mit Riddelliin und Lasiocarpin von Bedeutung $[19,25]$. Aus der Studie mit Riddelliin [19] wurde unter Anwendung eines Unsicherheitsfaktors von 100 und einem No-Observed-Adverse-Effect-Level(NOAEL) von 0,01 mg/ $\mathrm{kgKG} /$ Tag abgeleitet, dass unterhalb eines gesundheitsbasierten Richtwertes (Health Based Guidance Value, HBGV) von $0,1 \mu \mathrm{g} / \mathrm{kgKG} /$ Tag nichtneoplastische Schäden nicht zu erwarten sind [13, 26]. Bei einer Exposition unterhalb dieses Wertes ist auch nicht mit akuten Gesundheitsschäden zu rechnen. Hinsichtlich der krebserzeugenden Wirkung wurde die niedrigste $\mathrm{BMDL}_{10}$ von $0,073 \mathrm{mg} /$ kgKG/Tag zugrunde gelegt, die aus der Studie mit Lasiocarpin an männlichen Ratten [25] abgeleitet worden war, um den Margin of Exposure (MOE) für einzelne PA oder deren Summe abschätzen zu können. Dabei werden PA als Gruppe

Bundesgesundheitsbl 2017 -60:728-736 DOI 10.1007/s00103-017-2561-6

๑ Der/die Autor(en) 2017. Dieser Artikel ist eine Open-Access-Publikation.

B. Dusemund · I. M. C. M. Rietjens · A. Cartus · B. Schaefer · A. Lampen Pflanzliche Kontaminanten in Lebensmitteln. Vorkommen,
Wirkung und Risikobewertung

\section{Zusammenfassung}

Von den in der Lebensmittelkette vorkommenden Kontaminanten verursachen insbesondere diejenigen pflanzlicher Herkunft in den letzten Jahren zunehmende Besorgnis. Hierbei sind vielfältige unerwünschte Wirkungen in Betracht zu ziehen, die aufgrund potenziell vorliegender akuter, subchronischer oder chronischer Toxizität unterschiedliche gesundheitliche Risiken für den Verbraucher mit sich bringen können. Die zugrunde liegenden Mechanismen der Toxizität können Schwellenwerte bedingen oder aber, wie bei genotoxischen
Kanzerogenen, auf Interaktionen mit der DNA beruhen, für die kein Schwellenwert angenommen wird. Es wird ein Überblick über die hauptsächlichen, gegenwärtig relevanten Lebensmittelkontaminanten pflanzlichen Ursprungs gegeben und es werden ihre Wirkungsweisen und unerwünschten Effekte beschrieben.

Schlüsselwörter

Pyrrolizidinalkaloide - Tropanalkaloide . Opiumalkaloide $\cdot$ Tetrahydrocannabinol

\section{Plant-derived contaminants in food. Occurrence, effects and risk assessment}

\section{Abstract}

Among the various contaminants, the group of natural plant-derived substances in the modern food chain has been generating increasing concern in recent years. The adverse effects encountered may be diverse and pose risks of acute, subchronic or chronic toxicity. The underlying mechanisms of toxicity may be thresholded or be based on interactions with DNA, as for genotoxic carcinogens, for which the existence of a threshold cannot be assumed. This article gives an overview of the major plant-derived contaminants of present concern in the modern food chain and describes their mode of action and adverse effects.

\section{Keywords}

Pyrrolizidine alkaloids · Tropane alkaloids . Opium alkaloids - Tetrahydrocannabinol mit kumulativer Wirkung angesehen (z. B. [13, 18, 26]). Der MOE-Ansatz dient zur Abschätzung des Risikos von genotoxisch und kanzerogen wirkenden Substanzen. Dabei wird angenommen, dass für genotoxische Kanzerogene ein MOE von 10.000 oder höher im Hinblick auf die öffentliche Gesundheit als wenig bedenklich angesehen wird und deshalb als niedrige Priorität für Maßnahmen des Risikomanagements erachtet werden kann [27].

In seiner aktuellen Stellungnahme kommt das Bundesinstitut für Risikobewertung (BfR) zu dem Schluss, dass mit PA verunreinigte Kräutertees, einschließlich Rooibostee, schwarzer und grüner Tee sowie Honig die Hauptquellen sind, über die PA mit der Nahrung aufgenommen werden. Die in diesen Lebensmitteln enthaltenen PA-Mengen können sowohl für Kinder als auch für Erwachsene bei langfristiger Aufnahme gesundheitlich bedenklich sein. Ein akutes Gesundheitsrisiko besteht jedoch nicht [13]. Ähnliche Schlüsse sind aus einer aktuellen Stellungnahme der Europäischen Behörde für Lebensmittelsicherheit (EFSA) zur akuten und chronischen Exposition mit PA durch pflanzliche Lebensmittel zu ziehen, in der ebenfalls Tee- und Kräuterteeaufgüsse als die Hauptquellen für PA-Aufnahmen identifiziert wurden [28]. Die höchsten Schätzwerte für die akute und chronische Exposition ergaben sich hierbei für Kleinkinder. 


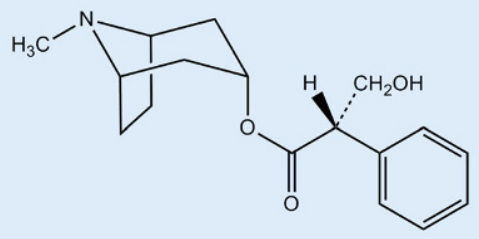

(-)-Hyoscyamin

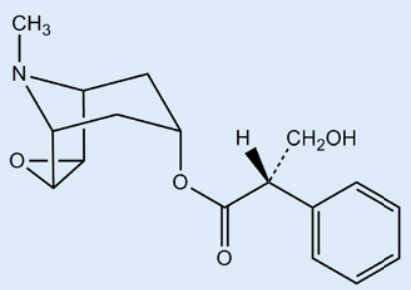

Scopolamin ((-)-Hyoscyin)

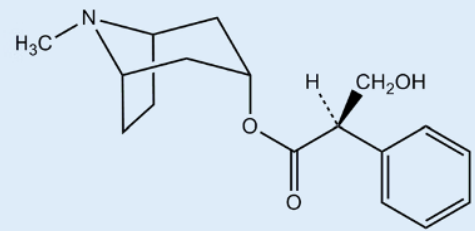

(+)-Hyoscyamin

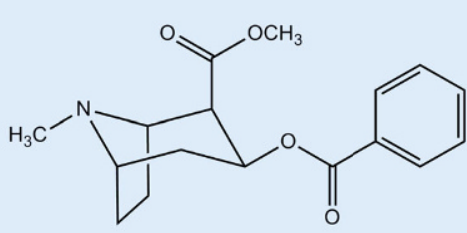

Cocain

Abb. 2 \ Strukturformeln wichtiger Tropanalkaloide. Das Racemat von (-)- und (+)-Hyoscyamin ist als Atropin bekannt

\section{Tropanalkaloide (TA)}

\section{Chemische und botanische Charakterisierung, Vorkommen}

Tropanalkaloide (TA) sind in verschiedenen Pflanzenfamilien wie den Solanaceae, Erythroxylaceae, Convolvulaceae, Brassicaceae und Euphorbiaceae verbreitet und bilden die Grundlage für das toxische Prinzip der Samen des Stechapfels (Datura stramonium), der Beeren der Tollkirsche (Atropa belladonna) sowie der Samen des Bilsenkrautes (Hyoscyamus niger), die als Kontaminanten von Lebensmitteln bekannt sind [29]. Typisch ist die Verunreinigung von Getreide (z. B. Hirse, Buchweizen) mit Samenkörnern von Bilsenkraut oder Stechapfel, sodass TA vielfach in getreidebasierten Lebensmitteln nachgewiesen wurden. In Untersuchungen von Mulder et al. [30] wurden die höchsten Konzentrationen an Gesamtalkaloiden - Summe aus (-)-Scopolamin und Atropin - aber für Kräutertees gemessen. Insgesamt wurden bisher mehr als 200 verschiedene TA in Pflanzen identifiziert. Einige dieser Alkaloide werden auch als Arzneimittelwirkstoffe eingesetzt, so z. B. (-)Scopolamin, (-)-Hyoscyamin, Atropin (ein Gemisch aus gleichen Anteilen (-)-
Hyoscyamin und (+)-Hyoscyamin) und Cocain (• Abb. 2). Auf Cocain, das als Lebensmittelkontaminante eine untergeordnete Rolle spielt, wird hier nicht weiter eingegangen (vgl. aber [31]).

\section{Gefährdungspotenzial, toxiko- logische und pharmakologische Daten}

Daten zur Toxizität der TA liegen nur in sehr begrenztem Umfang und vornehmlich für (-)-Hyoscyamin und (-)Scopolamin vor. Aktuelle Bewertungen beziehen sich daher nur auf diese beiden Alkaloide bzw. auf Atropin als das Racemat aus (-)-Hyoscyamin und (+)-Hyoscyamin [29, 32]. (-)-Hyoscyamin und (-)-Scopolamin kommen natürlich, z. B. als Hauptinhaltsstoffe in der Tollkirsche (Atropa belladonna) und in StechapfelArten (Datura spp.), vor. Vergiftungen durch Verzehr von Lebensmitteln, die mit Teilen dieser Pflanzen kontaminiert waren, sind beschrieben worden [29, 32]. Im Jahr 2003 wurden 73 Vergiftungsfälle aus Slowenien bekannt, wo Verbraucher mit Stechapfelsamen kontaminierte Buchweizenprodukte gegessen hatten. Innerhalb von $48 \mathrm{~h}$ wurden die für Tropanalkaloide typischen Intoxikationssymptome (trockener Mund, heiße rote Haut, Sehstörungen, Tachykardie, Harnverhalt, Ataxie, Sprachstörungen, Desorientierung und visuelle Halluzinationen) beobachtet. Der Buchweizen war mit 190 Samen von D. stramonium $/ \mathrm{kg}$ verunreinigt gewesen [33]. Die Exposition von 12 der betroffenen Personen wurde auf $0,7-138 \mu \mathrm{g} / \mathrm{kgKG}$ für (-)Hyoscyamin und $0,4-64 \mu \mathrm{g} / \mathrm{kgKG}$ für (-)-Scopolamin geschätzt [34].

(-)-Hyoscyamin/Atropin ${ }^{1}$ und (-)Scopolamin werden pharmazeutisch als Muscarinrezeptorantagonisten (Anticholinergika, Parasympatholytika) oral zur Therapie von Spasmen im MagenDarm-Kanal, der Gallenwege oder der Harnwege bzw. zur Prävention von Kinetosen (Bewegungskrankheiten, Reisekrankheit) eingesetzt [35-37]. Die niedrigste therapeutische orale Einzeldosis beträgt $1,4 \mu \mathrm{g} / \mathrm{kgKG}$ für (-)-Hyoscyamin, $6 \mu \mathrm{g} / \mathrm{kgKG}$ für Atropin (entsprechend ca. $3 \mu \mathrm{g} / \mathrm{kgKG}[-]-$ Hyoscyamin) und $2,5 \mu \mathrm{g} / \mathrm{kgKG}$ für (-)-Scopolamin. Als unerwünschte Wirkungen dieser Alkaloide bei niedrigster oraler therapeutischer Dosierung sind Verlangsamung der Herzfrequenz, Mundtrockenheit und verminderte Schweißabsonderung bekannt. In höheren therapeutischen oralen Dosen führen sie u. a. zur Herzfrequenzsteigerung und Mydriasis. Anders als (-)Hyoscyamin oder Atropin bewirkt (-)Scopolamin im Bereich oraler therapeutischer Dosierungen eine Depression des Zentralnervensystems (ZNS). Dosisbezogene Angaben zum Auftreten unerwünschter Wirkungen gelten nicht für Patienten mit Erkrankungen, für die Kontraindikationen oder Vorsichtsmaßnahmen bezüglich der Therapie mit TA beschrieben sind und die empfindlicher reagieren können als andere Patienten. Anwendungsbeschränkungen bezüglich einer oralen Therapie mit (-)-Hyoscyamin/Atropin oder (-)-Scopolamin bestehen auch während der Schwangerschaft oder Stillzeit. Beim Überschreiten des therapeutischen Dosisbereiches bewirken alle genannten Alkaloide ZNSStimulierung, psychotische Reaktionen und Halluzinationen. Erst bei massiver

1 Es wird davon ausgegangen, dass (-)-Hyoscyamin ungefähr die 2fache antimuscarine Aktivität von Atropin hat (EFSA 2013). 


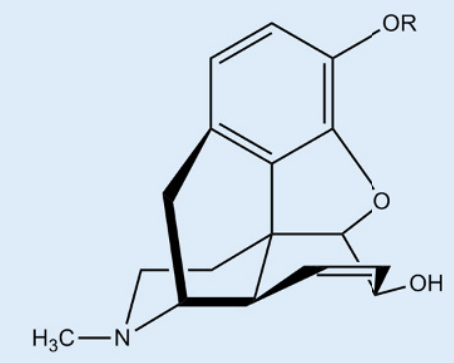

Morphin: $\mathrm{R}=\mathrm{H}$

Codein: $\mathrm{R}=\mathrm{CH}_{3}$

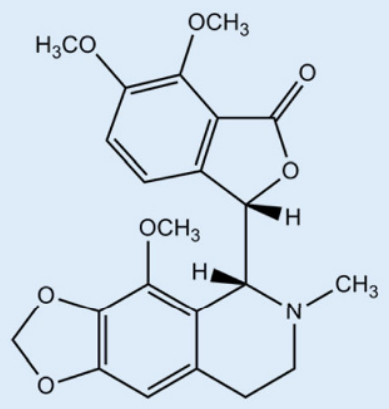

Noscapin

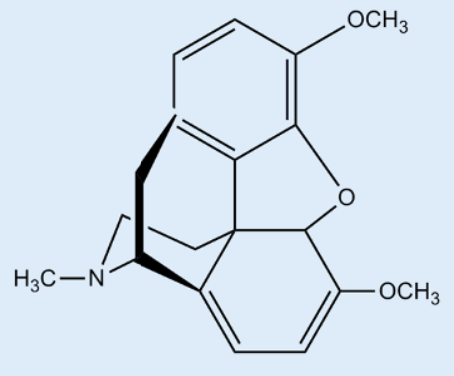

Thebain

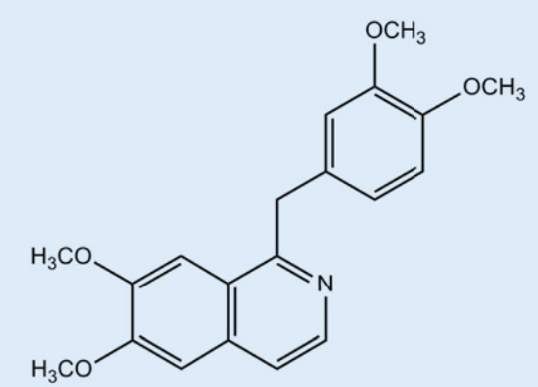

Papaverin

Abb. $3 \Delta$ Strukturformeln wichtiger Opiumalkaloide

Überdosierung oder schweren Vergiftungen schlägt die ZNS-Wirkung in eine Depression um und es kann der Tod durch Atemstillstand eintreten [29, 32].

\section{Risikocharakterisierung und -bewertung}

Die EFSA geht davon aus, dass bei der Risikobewertung von (-)-Hyoscyamin und (-)-Scopolamin die akuten pharmakologischen und toxikologischen Wirkungen im Vordergrund stehen und leitet daher eine Akute Referenzdosis (ARfD) für diese Substanzen ab. Die Ableitung basiert auf Beobachtungen an 20 gesunden jungen männlichen Erwachsenen bezüglich der Senkung der Herzfrequenz und ZNS-Effekten wie Benommenheit, Kopfschmerzen und Übelkeit [34]. Unter Zugrundelegung eines NOAEL von $0,16 \mu \mathrm{g} / \mathrm{kgKG}$ und Anwendung eines Unsicherheitsfaktors von 10 zur Berücksichtigung interindividueller Unterschiede wurde eine auf die Summe von (-)-Hyoscyamin und (-)Scopolamin bei Annahme äquivalenter
Potenz bezogene Gruppen-ARfD von $0,016 \mu \mathrm{g} / \mathrm{kgKG}$ ermittelt. Da TA nicht akkumulieren und keine Hinweise auf Genotoxizität oder chronische Toxizität vorliegen, würde eine auf der ARfD beruhende Risikoabschätzung auch Risiken bei chronischer Exposition mit einschließen. Die EFSA weist darauf hin, dass die Gruppen-ARfD zwei Größenordnungen unterhalb der niedrigsten therapeutischen Dosen für (-)-Hyoscyamin und (-)-Scopolamin liegt.

Auf Basis der verfügbaren limitierten Daten kommt die EFSA zu dem Schluss, dass die Gruppen-ARfD für die Tropanalkaloide Atropin, (-)-Hyoscyamin und (-)-Scopolamin bei Kleinkindern durch den Verzehr von Getreideprodukten um das bis zu 7 fache überschritten werden kann.

Das BfR hält die von der EFSA abgeleitete Gruppen-ARfD von $0,016 \mu \mathrm{g} / \mathrm{kgKG}$ für eine adäquate Basis für die Risikobewertung TA-kontaminierter Lebensmittel [32] und empfiehlt Maßnahmen, die sicherstellen, dass die TA-Aufnahmen auch bei Hochverzehr relevanter
Lebensmittelgruppen die ARfD nicht überschreiten. Dementsprechend sieht die Verordnung (EG) Nr. 1881/2006, zuletzt geändert durch die Verordnung (EU) 2016/239 der Kommission vom 19. Februar 2016, bei Getreidebeikost und anderer Beikost für Säuglinge und Kleinkinder, die Hirse, Sorghum, Buchweizen oder daraus gewonnene Erzeugnisse enthält, bereits Höchstgehalte von $1 \mu \mathrm{g}$ Atropin/kg und $1 \mu \mathrm{g}$ Scopolamin $/ \mathrm{kg}$ vor.

\section{Opiumalkaloide}

\section{Chemische und botanische Charakterisierung, Vorkommen}

Der in allen Erdteilen erfolgende Anbau von Schlafmohn (Papaver somniferum) dient der Samen- und Ölgewinnung für die Verwendung in Lebensmitteln sowie der Alkaloid- und Opiumherstellung [38]. Opium ist der durch Einschnitte der unreifen Kapseln ausgeflossene und auf der Kapseloberfläche eingetrocknete Milchsaft. Es enthält ca. 20-25 \% Alkaloide, von denen bisher etwa 50 Alkaloide in reiner Form isoliert wurden und insbesondere die mit Phenanthren- (Morphin, Codein, Thebain) oder Benzylisochinolinstruktur (Papaverin, Noscapin), deren Strukturformeln sich in $\bullet \mathbf{A b b} .3$ finden, toxikologisch relevant sind. Hauptalkaloid des Opiums ist das Morphin, das auch in größter Konzentration von meist $12 \%$ (je nach Herkunft 7-20\%) vorhanden ist. Die Konzentrationen der Nebenalkaloide im Opium betragen für Codein ca. $2 \%$ $(0,3-6 \%)$, Thebain ca. $0,5 \%(0,2-1 \%)$, Noscapin 5\% (2-12\%) und Papaverin ca. $1 \%(0,5-3 \%)[36,38]$.

Die Alkaloide werden in dem Latex (Milchsaft) synthetisiert, gespeichert und metabolisiert, der sich in anastomosierenden Milchröhren befindet. Diese durchziehen alle Teile der Pflanze und besonders dicht das Perikarp der Fruchtkapsel, jedoch nicht die Samen, die als einziges milchsaftloses Organ gelten [36, 38, 39]. Als Ursache für die teilweise hohen Opiatgehalte von Mohnsamen wurden daher insbesondere weniger geeignete Erntemethoden genannt. Durch letztere kann es zur Verunreinigung von Mohnsamen mit alkaloidhaltigen Kap- 
selbruchstücken und Latex kommen, sodass davon ausgegangen wird, dass die Opiumalkaloide nur als äußere Kontamination an den Mohnsamen haften. Dies wird auch aus Befunden abgeleitet, nach denen der Morphingehalt von Samen durch Waschen drastisch reduziert werden konnte [4, 40, 41]. Im Jahr 2004 wurde auf der Grundlage internationaler Daten über maximale Gehalte in Mohnsamen für Morphin von $620 \mu \mathrm{g} / \mathrm{g}$, für Codein von $57,1 \mu \mathrm{g} / \mathrm{g}$, für Noscapin von $230 \mu \mathrm{g} / \mathrm{g}$, für Papaverin von $67 \mu \mathrm{g} / \mathrm{g}$ und für Thebain von $41 \mu \mathrm{g} / \mathrm{g}$ berichtet [41]. Es ist bekannt, dass Verfahren der Lebensmittelverarbeitung, wie z. B. Waschen, Einweichen, Mahlen und Kochen, den Alkaloidgehalt von Mohnsamen verringern können. So lässt sich durch Waschen in Kombination mit Rösten eine Absenkung des Ausgangsmorphingehaltes von Mohnsamen um 98-100\% erreichen [42].

Von australischen Mohnsamen ist bekannt, dass er außer den genannten Opiumalkaloiden auch das Phenanthrenalkaloid Oripavin enthalten kann, ein Biosynthesevorläufer vom Morphin [40].

\section{Gefährdungspotenzial, toxiko- logische und pharmakologische Daten}

Relevant sind in diesem Zusammenhang vornehmlich Humandaten, die aus der arzneilichen Anwendung der Opiumalkaloide bekannt sind, sowie Erkenntnisse, die aus Vergiftungsfällen und Studien mit opiumalkaloidkontaminierten Mohnsamen stammen, wobei der Morphinwirkung die höchste Bedeutung zukommt $[4,40]$. Die diversen Morphinwirkungen auf das zentrale und periphere Nervensystem werden hauptsächlich durch $\mu$-Opiatrezeptoren vermittelt. Während auch die anderen Phenanthrenalkaloide mit $\mu$-Opiatrezeptoren reagieren, ist dies für die Benzoylchinolinalkaloide nicht bekannt [40].

Arzneilich wird Morphin hauptsächlich zur Behandlung starker Schmerzen eingesetzt. $\mathrm{Zu}$ den unerwünschten Wirkungen gehören u.a. bei moderater Dosierung Übelkeit, Erbrechen, Benommenheit, Veränderungen in kognitiven und sensorischen Fähigkeiten, Euphorie, Dysphorie, Halluzinationen, Mundtrockenheit, Miosis und Herz-KreislaufEffekte sowie bei höherer Dosierung Atemdepression, Kreislaufversagen und Konvulsionen. Der langfristige Einsatz kann zu Toleranzentwicklung sowie zu psychischer und physischer Abhängigkeit führen. Dabei schwankt die individuelle Empfindlichkeit für die arzneilich erwünschten und unerwünschten Wirkungen erheblich. Die niedrigste therapeutische Einzeldosis für die orale Einnahme von 1,9 mg Morphin, die bei einem Körpergewicht von $60 \mathrm{~kg}$ einer Dosis von $31,7 \mu \mathrm{g} / \mathrm{kgKG}$ entspricht wurde als Lowest Observed Effect Level (LOEL) betrachtet $[4,40]$.

Die für Vergiftungsfälle mit opiumalkaloidhaltigen Mohnsamen beschriebenen Symptome stimmen mit den aus der pharmazeutischen Anwendung beschriebenen adversen Effekten überein. So stellte eine Verbraucherin nach Verzehr eines Nudelgerichtes, das mit gemahlenem Mohn bestreut worden war, ein „schwummriges Gefühl“ im Kopffest, musste sich übergeben und beobachtete am nächsten Tag ein Katergefühl. Die Betroffene hatte ca. $75 \mathrm{~g}$ Blaumohn, der $210 \mu \mathrm{g}$ Morphin/g und $39 \mu \mathrm{g}$ Codein/g enthielt, zu sich genommen, was Einnahmedosen von $16 \mathrm{mg}$ Morphin und $3 \mathrm{mg}$ Codein entspricht. Die Mohnprobe wurde (im Sinne von Art. 14 Abs. 2a und Abs. 4 der VO (EG) 178/2002) von der Lebensmittelüberwachung als geeignet beurteilt, die Gesundheit des Verbrauchers $\mathrm{zu}$ beeinträchtigen [4]. In einem weiteren Vergiftungsfall, in dem Backmohn, nicht der Bestimmung als Lebensmittel entsprechend, als „Schlafmittel“ verabreicht wurde, erlitt ein 6 Wochen alter Säugling eine schwere Atemdepression und musste intensivmedizinisch behandelt werden. Die Mutter hatte ihm $75 \mathrm{ml}$ einer Milchzubereitung gegeben, die sie nach Abseihen aus einer Mischung von $200 \mathrm{~g}$ Mohnsamen und $500 \mathrm{ml}$ Milch erhalten hatte. Gemäß den Untersuchungen betrug der Morphinspiegel im Serum des Säuglings am Folgetag 4,3 ng/ml. Im verwendeten Mohnsamen waren 0,1\% Morphin und 0,003\% Codein nachgewiesen worden [4].

\section{Risikocharakterisierung und -bewertung}

Basierend auf dem aus dem Arzneimittelbereich bekannten LOEL, leitete das BfR im Jahr 2005 unter Anwendung eines Unsicherheitsfaktors von 5 eine „vorläufige maximale tägliche Aufnahmemenge“ für Morphin von 6,3 $\mu \mathrm{g}$ Morphin/kgKG/ Tag ab, die bei einer mohnsamenhaltigen Mahlzeit pro Tag oder über den Tag verteilt nicht überschritten werden sollte [4]. Auf den gleichen LOEL Bezug nehmend, legte die EFSA unter Anwendung eines Unsicherheitsfaktors von 3 eine ARfD von $10 \mu \mathrm{g}$ Morphin/kgKG fest und kam auf der Basis aktueller Expositionsschätzungen zu dem Schluss, dass insbesondere Konsumenten von Lebensmitteln, die große Mengen von Mohnsamen enthalten, die ARfD überschreiten können.

\section{Delta-9-Tetrahydrocannabinol (THC)}

\section{Chemische und botanische Charakterisierung, Vorkommen}

Delta-9-Tetrahydrocannabinol (THC, - Abb. 4) ist der rauscherzeugende Hauptinhaltsstoff der Hanfpflanze (Cannabis sativa). Hanf spielte über Jahrtausende nicht nur als Faserpflanze, sondern auch als Nahrungslieferant in vielen Gegenden der Welt eine bedeutende Rolle. Er tritt in verschiedenen Kulturformen auf, die unter dem Speziesnamen Cannabis sativa zusammengefasst werden. Der gewöhnliche Hanf (Cannabis sativa L. non indica) liefert normalerweise keine rauscherzeugenden Drogen, sondern wird zur Gewinnung der Hanffasern angebaut und enthält im Samen u. a. bis zu 35 \% fettes Öl mit ca. 70 \% Linolsäureund ca. $15 \%$ Ölsäureglyceriden sowie ca. $25 \%$ Protein. In der Bundesrepublik Deutschland ist der Anbau von Hanf nur mit einer entsprechenden Genehmigung erlaubt, die u. a. das verwendete Saatgut genau vorschreibt. Neben dem Gebrauch als Faser- und Ölpflanze finden einzelne der im Hanf enthaltenen Cannabinoide auch als Arzneimittel und z. T. illegal als Rauschmittel (Marihuana, Haschisch) Verwendung [38]. Die THCKonzentration überschreitet bei Hanf 


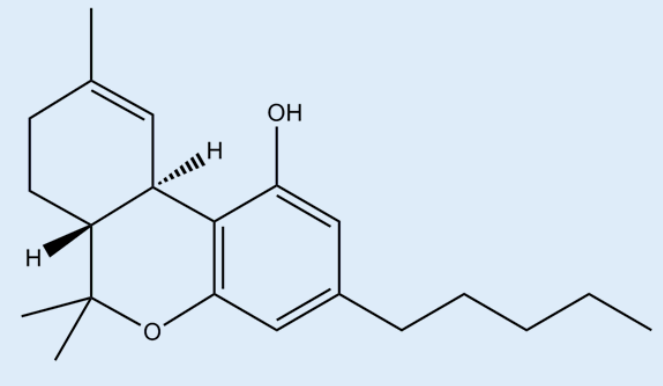

Delta-9-tetrahydrocannabinol
Abb. $4<$ Strukturformel von Delta-9Tetrahydrocannabinol (THC) vom Fasertyp 0,4\% nicht und kann bei Hanf vom Drogentyp bis zu $5 \%$ betragen $[43,44]$.

Aus Hanf wurden über 400 Inhaltsstoffe isoliert. Als Hauptinhaltsstoffe angesehen werden die phenolischen, lipophilen Cannabinoide mit dem eigentlichen psychotrop wirksamen Prinzip des Hanfs, dem THC, das im Hanf vornehmlich in Form seiner inaktiven Vorstufe, der Delta-9-Tetrahydrocannabinolsäure (2-COOH-Delta-9-Tetrahydrocannabinol; THC-A) vorliegt, wobei die höchsten Konzentrationen im Harz der Trichome (Drüsenhaare) der Blütenstände weiblicher Pflanzen gefunden werden. THC-A decarboxyliert während der Lagerung, Alterung und Erhitzen des pflanzlichen Materials zu THC. Da der Hanfsamen per se kein THC/THC-A enthält, gilt THC in Hanfsamen und dem daraus gewonnenen Hanfsamenöl als Kontaminante. Es wird davon ausgegangen, dass es ernteprozessbedingt durch den Samen anhaftende andere Pflanzenteile der Hanfpflanze in Hanfsamen und Hanfsamenöl gelangt [45-47].

\section{Gefährdungspotenzial, toxiko- logische und pharmakologische Daten}

Im Tierversuch wurden u. a. hormonelle Störungen und immunschwächende Effekte beschrieben. Aussagekräftiger sind aber Humandaten. Vier Vergiftungsfälle mit typischen zentralnervösen Symptomen (Übelkeit, Erbrechen, Schwindel, Wahrnehmungsstörungen) nach Verzehr eines mit THC kontaminierten Hanfsamenöles sind beschrieben worden [3]. Auch wurde beim Menschen in verschiedenen Studien nach oraler Aufnahme eine Vielzahl unerwünschter Wirkungen beobachtet. In klinischen Untersuchungen, in denen HIV-Patienten zur Appetitsteigerung über mehrere Wochen THC erhielten, wurden bereits bei Tagesdosen von 2,5 mg THC Wirkungen auf das Herz-Kreislauf-System (Tachykardie) und auf das zentrale Nervensystem (Schwindel, Bewusstseinsstörungen, Sedierung, Müdigkeit, Übelkeit, Euphorie und Angstgefühle) beobachtet [48, 49]. Die tägliche Dosis von $2,5 \mathrm{mg}$ THC, entsprechend $0,036 \mu \mathrm{g} / \mathrm{kgKG} / \mathrm{Tag}$ $(\mathrm{KG}=70 \mathrm{~kg})$ wurde daher als der Lowest Observed Adverse Effect Level (LOAEL) angesehen.

\section{Risikocharakterisierung und -bewertung}

Auf den aus den Studien von Beal et al. [48, 49] abgeleiteten LOAEL von $2,5 \mathrm{mg}$ THC/Person/Tag wurde unter Einbeziehung von Unsicherheitsfaktoren in den Risikobewertungen des Bundesinstitutes für gesundheitlichen Verbraucherschutz und Veterinärmedizin (BgVV; Vorgängerinstitut des BfR) und von der EFSA Bezug genommen $[50,51]$. So empfahl das BgVV im Jahre 1997 in einer vorläufigen Abschätzung beim Verzehr von Lebensmitteln eine tägliche Aufnahmemenge an THC von 1-2 $\mu \mathrm{g} / \mathrm{kgKG}$ nicht $\mathrm{zu}$ überschreiten. Ähnlich leitete EFSA in der unlängst für Lebensmittel tierischer Herkunft durchgeführten Risikobewertung eine ARfD von $1 \mu \mathrm{g}$ THC/kgKG ab [51]. Basierend auf den genannten Humandaten empfahl das BgVV Richtwerte für Gesamt-THC (THC und THC-A), die in verzehrsfertigen hanfhaltigen Lebensmitteln nicht überschritten werden sollten: $5 \mu \mathrm{g} / \mathrm{kg}$ für nichtalkoholische und alkoholische Getränke, $5000 \mu \mathrm{g} / \mathrm{kg}$ für Speiseöle, $150 \mu \mathrm{g} / \mathrm{kg}$ für alle anderen Lebensmittel $[45,52]$.

\section{Ausblick}

Den vorgestellten Risikocharakterisierungen von Kontaminanten botanischen Ursprungs in Lebensmitteln ist gemein, dass sie angesichts der komplexen $\mathrm{Zu}$ sammensetzung pflanzlichen Materials auf der Bewertung des Potenzials der toxikologisch relevanten Hauptinhaltsstoffe bestehen, zu denen vielfach $\mathrm{Hu}$ mandaten aufgrund von arzneilichen Anwendungen oder von Vergiftungsfällen vorliegen. Die Nebenalkaloide sind, wie z. B. das Oripavin in Mohnsamen bestimmter Provenienz, nur ungenügend toxikologisch charakterisiert. Künftige Forschung dürfte darauf zielen, diese Wissenslücken $\mathrm{zu}$ schließen, um dem bestehenden Ziel besser gerecht werden zu können, Interaktionen verschiedener, gemeinsam als Verunreinigung auftretender Alkaloide spezifischer in die Risikobewertung einzubeziehen. Hierzu sollten auch die molekularen Wirkmechanismen der Nebenalkaloide genauer verstanden werden, um diese möglicherweise in die Risikobewertung besser einbeziehen zu können.

Typisch für pflanzliche Kontaminanten ist auch, dass sich Ansätze für Vermeidungsstrategien aus Analysen von Anbau-, Herstellungs- und Verarbeitungsbedingungen der betroffenen pflanzlichen Rohmaterialien ergeben. Um die gesundheitliche Unbedenklichkeit pflanzlicher Lebensmittel garantieren zu können, werden bestehende Anstrengungen, dem zunehmenden Wissensstand entsprechend, künftig verstärkt werden müssen. Dies betrifft den Ausbau und die Standardisierung analytischer Methoden wie auch Maßnahmen von Lebensmittelüberwachung und Herstellerseite. Es ist zu erwarten, dass weitere Höchstmengenfestlegungen in Verordnung (EG) Nr. 1881/2006 für die genannten Alkaloide in relevanten Lebensmitteln als Risikomanagementmaßnahmen folgen. 


\section{Korrespondenzadresse}

\section{Dr. B. Dusemund}

Bundesinstitut für Risikobewertung

Berlin, Deutschland

birgit.dusemund@bfr.bund.de

\section{Einhaltung ethischer Richtlinien}

Interessenkonflikt. B. Dusemund, I.M.C.M. Rietjens, A. Cartus, B. Schaefer und A. Lampen geben an, dass kein Interessenkonflikt besteht.

Dieser Beitrag beinhaltet keine von den Autoren durchgeführten Studien an Menschen oder Tieren.

Open Access. Dieser Artikel wird unter der Creative Commons Namensnennung 4.0 International Lizenz (http://creativecommons.org/licenses/by/4.0/deed. de) veröffentlicht, welche die Nutzung, Vervielfältigung, Bearbeitung, Verbreitung und Wiedergabe in jeglichem Medium und Format erlaubt, sofern Sie den/die ursprünglichen Autor(en) und die Quelle ordnungsgemäßnennen, einen Link zur Creative Commons Lizenz beifügen und angeben, ob Änderungen vorgenommen wurden.

\section{Literatur}

1. Xenophon (370 v. Chr./1999) Anabasis. Reclam, Ditzingen

2. BfR (Bundesinstitut für Risikobewertung) (2010) Vergiftungsfälle durch Grayanotoxine in Rhododendron-Honigen aus der türkischen Schwarzmeerregion. Stellungnahme Nr. 043/2010 des BfR vom 3. September 2010

3. Meier H, Vonesch HJ (1997) Cannabis-Intoxikation nach Salatgenuss. Swiss Med Wkly 127:214-218

4. BfR (Bundesinstitut für Risikobewertung) (2006) BfR empfiehlt vorläufige maximale tägliche Aufnahmemenge und einen Richtwert für Morphin in Mohnsamen. Gesundheitliche Bewertung Nr. 012/2006 des BfR vom 27. Dezember 2005

5. Schrenk D (2004) Chemische Lebensmittelkontaminanten. Bundesgesundheitsblatt Gesundheitsforschung Gesundheitsschutz 47:841-847

6. Rietjens IMCM, Martena MJ, Boersma MG, Spiegelenberg W, Alink GM (2005) Molecular mechanisms of toxicity of important food-borne phytotoxins. Mol Nutr Food Res 49:131-158

7. Speijers G, Bottex B,Dusemund B et al (2010) Safety assessment of botanicals and botanical preparations used as ingredients in food supplements: testing an European Food Safety Authority-tiered approach. Mol Nutr Food Res 54:175-185

8. Koleva II, van Beek TA, Soffers AEMF, Dusemund B, Rietjens IMCM (2012) Alkaloids in the human food chain - natural occurrence and possible adverse effects. Mol Nutr Food Res 56:30-52

9. Dusemund B, Appel K-E, Lampen A (2010) BfR risk assessment of alkaloids as ingredients and contaminants of food: quinine, opium alkaloids, and senecio pyrrolizidine alkaloids. In: DFG Senate Commission on Food Safety (SKLM) (Hrsg) Risk assessment of phytochemicals in food. Wiley-VCH, Weinheim, S1382-1390

10. Dusemund B (2014) Risk assessment of food components with botanical origin. In: Reichl F-X,
SchwenkM(Hrsg) Regulatory toxicology. Springer, Berlin

11. WHO (World Health Organization: International Programme on Chemical Safety (IPCS)) (1988) Pyrrolizidine alkaloids. Environ Health Criter 80

12. Codex Alimentarius Commission (2011) Discussion paper on pyrrolizidine alkaloids. Joint FAO/WHO Food Standards Programme. Codex Committee on Contaminants in Foods 5th Session, The Hague, The Netherlands, 21-25 March 2011.

13. BfR (Bundesinstitut für Risikobewertung) (2016) Pyrrolizidinalkaloide: Gehalte in Lebensmitteln sollen nach wie vor so weit wie möglich gesenkt werden. Stellungnahme Nr. 030/2016 des BfR vom 28. September 2016

14. BfR (Bundesinstitut für Risikobewertung) (2007) Salatmischung mit Pyrrolizidinalkaloid-haltigem Greiskraut verunreinigt. Stellungnahme 028/2007 des BfR vom 10. Januar 2007

15. BfR (Bundesinstitut für Risikobewertung) (2013) Pyrrolizidinalkaloide in Kräutertees und Tees. Stellungnahme 018/2013 des BfR vom 5. Juli 2013

16. Mulder PPJ, Sánchez PL, These A, Preiss-Weigert A, Castellari M (2015) Occurrence of pyrrolizidine alkaloids in food. Efsa Support Publ EN-859:1-114

17. BfR (Bundesinstitut für Risikobewertung) (2011) Analytik und Toxizität von Pyrrolizidinalkaloiden sowie eine Einschätzung des gesundheitlichen Risikos durch deren Vorkommen in Honig. Stellungnahme Nr. 038/2011 des BfR vom 11. August 2011 ergänzt am 21. Januar 2013

18. EFSA (European Food Safety Authority: Scientific Panel on Contaminants in the Food Chain (CONTAM)) (2011) Scientific opinion on pyrrolizidine alkaloids in food and feed. EFSA J 9(11):2406

19. NTP (U.S. National Toxicology Program) (2003) NTP Technical Report on the toxicology and carcinogenesis studies of Riddelliine (CASNo. 23246-96-0) in $\mathrm{F} 344 / \mathrm{N}$ rats and $\mathrm{B} 6 \mathrm{C} 3 \mathrm{~F} 1$ mice (gavage studies). NTP Technical Report TR 508, NIH Publication No. 03-4442

20. Allgaier C, Franz S (2015) Risk assessment on the use of herbal medicinal products containing pyrrolizidine alkaloids. Regul Toxicol Pharmacol 73:494-500

21. WHO (World Health Organization: International Agency for Research on Cancer (IARC)) (1983) Some food additives, feed additives and naturally occurring substances. IARC Monogr Eval Carcinog Risks Hum 31:1-291

22. WHO (World Health Organization: International Agency for Research on Cancer (IARC)) (1987) Overall evaluations of carcinogenicity: an updating of IARCMonographs Volumes 1 to 42. IARCMonogr Eval Carcinog Risks Hum (Supplement 7):1-440

23. WHO (World Health Organization: International Agency for Research on Cancer (IARC)) (2002) Some traditional herbal medicines, some mycotoxins, naphthalene and styrene. IARC Monogr Eval Carcinog Risks Hum 82:1-556

24. Fu PP, Xia Q, Lin G, Chou MW (2004) Pyrrolizidine alkaloids - genotoxicity, metabolism enzymes, metabolic activation, and mechanisms. Drug MetabRev 36:1-55

25. NCl (National Cancer Institute) (1978) Bioassay of lasiocarpine for possible carcinogenicity CAS No. 303-34-4. Carcinogenesis. Technical Report Series NCl-CG-TR-39

26. FSA (Food Standards Agency: Committee on Toxicity of Chemicals in Food CPatEC) (2008) COT statement on pyrrolizidine alkaloids in food

27. EFSA (European Food Safety Authority) (2005) Opinion of the Scientific Committee on a request from EFSA related to a harmonised approach for risk assessment of substances which are both genotoxic and carcinogenic (Request No EFSA-Q2004-020 adopted on 18 October 2005). EFSA J 282:1-31

28. EFSA (European Food Safety Authority) (2016) Dietary exposure assessment to pyrrolizidine alkaloids in the European population. EFSA J 14(8):4572

29. EFSA (European Food Safety Authority: Scientific Panel on Contaminants in the Food Chain (CONTAM)) (2013) Scientific opinion on tropane alkaloids in food and feed. EFSA J 11(10):3386

30. Mulder PPJ, de Nijs M, Castellari M et al (2016) Occurrence of tropane alkaloids in food. EFSA Support Publ 13:EN-1140

31. BfR (Bundesinstitut für Risikobewertung) (2009) Kein Gesundheitsrisiko durch den Cocaingehalt in Red Bull Simply Cola Gesundheitliche Bewertung Nr. 020/2009 des BfR vom 27. Mai 2009

32. BfR (Bundesinstitut für Risikobewertung) (2013) Hohe Tropanalkaloidgehalte in Getreideprodukten: Bei Menschen mit Herzproblemen sind gesundheitliche Beeinträchtigungen möglich. Stellungnahme Nr. 035/2014 des BfR vom 13. November 2013

33. Perharic L (2005) Mass tropane alkaloid poisoning due to buckwheat flour contamination. Clin Toxicol 43:413

34. Perharic L, Juvan KA, Stanovnik L (2013) Acute effects of a low-dose atropine/scopolamine mixture as a food contaminant in human volunteers. J Appl Toxicol 33:980-990

35. Brown JH, Taylor P (2006) Muscarinic receptor agonists and antagonists. In: Brunton LL, Lazo JS, Parker KL (Hrsg) Goodman \& Gilman's the pharmacological basis of therapeutics, 11. Aufl. McGraw-Hill Medical Publishing Division, New York

36. Bracher F, Heisig P, Langguth $P$ et al (2016) Kommentar zum Europäischen Arzneibuch. Wissenschaftliche Verlagsgesellschaft, Stuttgart

37. Martindale (2017) The complete drug reference. https://www.medicinescomplete.com/mc/ martindale/2009/.Zugegriffen:20.01.2017

38. Hager ROM (2006) Hagers Enzyklopädie der Arzneistoffe und Drogen. Springer, Heidelberg

39. Hänsel R, Sticher O (2007) Pharmakognosie Phytopharmazie. Springer, Heidelberg

40. EFSA (European Food Safety Authority: Scientific Panel on Contaminants in the Food Chain (CONTAM)) (2011) Scientific opinion on the risks for public health related to the presence of opium alkaloids in poppy seeds. EFSA J 9(11):2405

41. Rochholz G, Westphal F, Wiesbrock UO, Schütz HW (2004) Opiat-Nachweis in Urin, Blut und Haaren nach Verzehr mohnsamenhaltiger Backwaren. Blutalkohol 41:319-329

42. General J, Unbehend G, Lindhauer MG, Kniel B, Moser M (2007) Untersuchungen zur Reduzierung von Morphin in Mohnsamen und Mohngebäcken mit praktikablen technologischen Maßnahmen. Getreidetechnologie 61:36-42

43. Avico U, Pacifici R, Zuccaro P (1985) Variations of tetrahydrocannabinol content in cannabis plants to distinguish thefibre-typefrom drug-typeplants Bull Narc 37:61-65

44. Taschan H (1999) Hanfhaltige Lebensmittel: Psychedelische Lebensmittel, neuartige Lebensmittel oder Rauschmittel? aid-Verbraucherdienst 44:144-148

45. Heinke V, Lampe U, Goehlsdorf K, Fuerst P, Boess C, Dusemund B (2002) Entwicklung von Methoden zur Bestimmung von Delta-9-Tetrahydrocan- 


\section{Leitthema}

nabinol in hanfhaltigen Lebensmitteln. BgVV 2002(07): $1-77$

46. Mölleken H, Husmann H (1997) Cannabinoids in seed extracts of cannabis sativa cultivars. $J$ Int Hemp Assoc 4:75-79

47. Zoller O, Rhyn P, Zimmerli B (2000) High-performance liquid chromatographic determination of delta9-tetrahydrocannabinol and the corresponding acid in hemp containing foods with special regard to the fluorescence properties of delta9-tetrahydrocannabinol. J Chromatogr A 872:101-110

48. Beal JE, Olson R, Laubenstein L et al (1995) Dronabinol as a treatment for anorexia associated with weight loss in patients with AIDS. J Pain Symptom Manage 10:89-97

49. Beal JE, Olson R, Lefkowitz L et al (1997) Long-term efficacy and safety of dronabinol for acquired immunodeficiency syndrome-associated anorexia.J Pain Symptom Manage 14:7-14

50. BgVV (Bundesinstitut für Gesundheitlichen Verbraucherschutz und Veterinärmedizin) (1997) Einsatz von Hanf in Lebensmitteln kann gesundheitlich problematisch sein. BgVV-Pressedienst 26/1997, 22.10.1997

51. EFSA (European Food Safety Authority: Scientific Panel on Contaminants in the Food Chain (CONTAM)) (2015) Scientific opinion on the risks for human health related to the presence of tetrahydrocannabinol (THC) in milk and other food of animal origin. EFSAJ 13(6):4141

52. BgVV (Bundesinstitut für Gesundheitlichen Verbraucherschutz und Veterinärmedizin) (2000) Richtwerte für THC in hanf-haltigen Lebensmitteln empfohlen. BgVV-Pressedienst 07/2000 vom 16.03.2000 